\title{
Global Robust Stability of Switched Interval Neural Networks with Discrete and Distributed Time-Varying Delays of Neural Type
}

\author{
Huaiqin Wu, Ning Li, Kewang Wang, Guohua Xu, \\ and Qiangqiang Guo \\ Department of Applied Mathematics, Yanshan University, Qinhuangdao 066001, China \\ Correspondence should be addressed to Huaiqin Wu, huaiqinwu@ysu.edu.cn
}

Received 24 May 2011; Accepted 6 July 2011

Academic Editor: Zidong Wang

Copyright (C) 2012 Huaiqin Wu et al. This is an open access article distributed under the Creative Commons Attribution License, which permits unrestricted use, distribution, and reproduction in any medium, provided the original work is properly cited.

By combing the theories of the switched systems and the interval neural networks, the mathematics model of the switched interval neural networks with discrete and distributed timevarying delays of neural type is presented. A set of the interval parameter uncertainty neural networks with discrete and distributed time-varying delays of neural type are used as the individual subsystem, and an arbitrary switching rule is assumed to coordinate the switching between these networks. By applying the augmented Lyapunov-Krasovskii functional approach and linear matrix inequality (LMI) techniques, a delay-dependent criterion is achieved to ensure to such switched interval neural networks to be globally asymptotically robustly stable in terms of LMIs. The unknown gain matrix is determined by solving this delay-dependent LMIs. Finally, an illustrative example is given to demonstrate the validity of the theoretical results.

\section{Introduction}

In the past few decades, neural networks have been a subject of intense research activities due to their wide applications in different areas such as image processing, pattern recognition, associative memory, and combinational optimization. A fundamental problem is the stability which is the prerequisite to ensure that the developed neural network can work [1-40]. In hardware implementation of the neural networks, time delay is inevitably encountered and is usually time varying due to the finite switching speed of amplifiers. It is known that time delay is often the main cause for instability and poor performance of neural networks. Moreover, due to unavoidable factors, such as modeling error, external perturbation, and parameter fluctuation, the neural networks model certainly involves uncertainties such as perturbations and component variations, which will change the stability of neural networks. 
To analyze uncertainty of neural networks, one reasonable method is to assume parameters in certain intervals. Therefore, it is of great importance to study the global robust stability of interval neural networks with time-varying delay. Recently, some sufficient conditions for the global robust stability of interval neural networks with time-varying delays and parametric uncertainties have been obtained in terms of LMIs [1-9].

Since neural networks usually have a spatial extent, there is a distribution of propagation delays over a period of time. In these circumstances, the signal propagation is not instantaneous and cannot be modeled with discrete delays, and a more appropriate way is to incorporate continuously distributed delays in neural network model. On the other hand, in certain physical systems, mathematical models have been described by some functional differential equations of neutral type, which depend on the delays of state and state derivative. In practice, neutral type phenomenon always appear in studies of automatic control, chemical reactors, population ecology, heat exchanges, microwave oscillators, and so on. Hence, the stability for neutral type neural networks with time-varying delay has been also considered in the recent years [10-18].

A class of hybrid systems has attracted significant attention because it can model several practical control problems that involve the integration of supervisory logic-based control schemes and feedback control algorithms. As a special class of hybrid systems, switched systems are regarded as nonlinear systems, which are composed of a family of continuous-time or discrete-time subsystems and a rule that orchestrates the switching between the subsystems. Recently, switched neural networks, whose individual subsystems are a set of neural networks, have found applications in fields of high-speed signal processing, artificial intelligence, and gene selection in a DNA microarray analysis [19-21]. Therefore, some researchers have studied the stability issues for switched neural networks [22-27]. In [22], based on the Lyapunov-Krasovskii method and LMI approach, some sufficient conditions were derived for global robust exponential stability of a class of switched Hopfield neural networks with time-varying delay under uncertainty. In [23], by combining CohenGrossberg neural networks with an arbitrary switching rule, the mathematical model of a class of switched Cohen-Grossberg neural networks with mixed time-varying delays were established, and the robust stability for such switched Cohen-Grossberg neural networks was analyzed. In [24], by employing nonlinear measure and LMI techniques, some new sufficient conditions were obtained to ensure global robust asymptotical stability and global robust stability of the unique equilibrium for a class of switched recurrent neural networks with time-varying delay. In [25], authors investigated a large class of switched recurrent neural networks with time-varying structured uncertainties and time-varying delay; some delaydependent robust periodicity criteria guaranteeing the existence, uniqueness, and global asymptotic stability of periodic solution for all admissible parametric uncertainties were devised by taking free weighting matrices and LMIs. In [26], based on multiple Lyapunov functions method and LMI techniques, the authors presented some sufficient conditions in terms of LMIs which guarantee the robust exponential stability for uncertain switched Cohen-Grossberg neural networks with interval time-varying delay and distributed timevarying delay under the switching rule with the average dwell time property.

It should be noted that, in the above literature, almost all results treated of the robust stability for switched neural networks with norm-bounded uncertainty. However, as it well known that there are two forms of parametric uncertainties, namely the interval uncertainty and the norm-bounded uncertainty. To the best of our knowledge, up to now, there are few researchers to deal with the global robust stability for switched neural networks with the interval uncertainty, despite its potential and practical importance. 
Motivated by the preceding discussion, the aim of this paper is to present a new class of the switched interval neural networks with discrete and distributed time-varying delays of neural type under interval parameter uncertainties by integrating the theory of switched systems with neural networks. By constructing a suitable Lyapunov-Krasovskii functional and employing Jensens inequality, a delay-dependent criterion will be derived such that the proposed switched interval neural networks are globally robustly asymptotically stable. The proposed criterion is represented in terms of LMIs, which can be solved efficiently by using recently developed convex optimization algorithms [28].

The rest of this paper is organized as follows. In Section 2, the model formulation and some preliminaries are given. The main result are stated in Section 3. In Section 4, a numerical example is presented to demonstrate the validity of the proposed results. Some conclusions are made in Section 5.

Notations. Throughout this paper, and $R$ denotes the set of real numbers, $R^{n}$ denotes the $n$-dimensional Euclidean space, $R^{m \times n}$ denotes the set of all $m \times n$ real matrices. For any matrix $A, A^{T}$ denotes the transpose of $A . A>0(A<0)$ means that $A$ is a positive definite (negative definite). Given the column vectors $x=\left(x_{1}, \ldots, x_{n}\right)^{T}, y=\left(y_{1}, \ldots, y_{n}\right)^{T} \in R^{n}$, and $x^{T} y=$ $\sum_{i=1}^{n} x_{i} y_{i} \dot{x}(t)$ denotes the derivative of $x(t)$, and $*$ represents the symmetric form of matrix.

\section{Neural Network Model and Preliminaries}

Consider the interval neural network model with discrete and distributed time-varying delays of neutral type described by the system of differential equations in the form

$$
\begin{array}{r}
\dot{x}(t)=-A x(t)+W_{1} g(x(t))+W_{2} g(x(t-\tau(t)))+W_{3} \int_{t-\tau(t)}^{t} g(x(s)) d s+W_{4} \dot{x}(t-\mu(t))+u, \\
A \in A_{l}, \quad W_{k} \in W_{l}^{(k)}, \quad k=1,2,3,4,
\end{array}
$$

where $x(t)=\left(x_{1}(t), \ldots, x_{n}(t)\right)^{T} \in R^{n}$ denotes the state vector associated with $n$ neurons; $g(x)=\left(g_{1}\left(x_{1}\right), \ldots, g_{n}\left(x_{n}\right)\right)^{T}: R^{n} \rightarrow R^{n}$ is a vector-valued neuron activation function; $u=\left(u_{1}, \ldots, u_{n}\right)^{T}$ is a constant external input vector; $\tau(t)$ denotes the discrete and distributed time-varying delays $\mu(t)$ represents neutral time-varying delays; $A=\operatorname{diag}\left(a_{1}, \ldots, a_{n}\right)$ is $n \times n$ constant diagonal matrices; $a_{i}>0, i=1, \ldots, n$, are the neural self-inhibitions; $W_{k}=\left[w_{i j}^{(k)}\right] \in$ $R^{n \times n}, k=1,2,3,4$, are the connection weight matrices; $A_{l}=[\underline{A}, \bar{A}]=\left\{A=\operatorname{diag}\left(a_{i}\right): 0<\underline{a}_{i} \leq\right.$ $\left.a_{i} \leq \bar{a}_{i}, i=1,2, \ldots, n\right\}, W_{l}^{(k)}=\left[\underline{W}_{k}, \bar{W}_{k}\right]=\left\{W_{k}=\left[w_{i j}^{(k)}\right]: \underline{w}_{i j}^{(k)} \leq w_{i j}^{(k)} \leq \bar{w}_{i j}^{(k)}, i, j=\right.$ $1,2, \ldots, n\}$ with $\underline{A}=\operatorname{diag}\left(\underline{a}_{1}, \underline{a}_{2}, \ldots, \underline{a}_{n}\right), \bar{A}=\operatorname{diag}\left(\bar{a}_{1}, \bar{a}_{2}, \ldots, \bar{a}_{n}\right), \underline{W}_{k}=\left[\underline{w}_{i j}^{(k)}\right]_{n \times n}, \bar{W}_{k}=$ $\left[\bar{w}_{i j}^{(k)}\right]_{n \times n}$.

Throughout this paper, the following assumptions are made on the activation functions $g_{j}, j=1,2, \ldots, n$, the discrete and distributed time-varying delay $\tau(t)$, and the neutral time-varying delay $\mu(t)$,

$$
\begin{aligned}
& \left(\mathscr{L}_{1}\right):\left|g_{j}\left(x_{1}\right)-g_{j}\left(x_{2}\right)\right| \leq k_{j}\left|x_{1}-x_{2}\right|, x_{1}, x_{2} \in R, j=1,2, \ldots, n, \\
& \left(\mathscr{L}_{2}\right): 0 \leq \tau(t) \leq \tau_{N}, \dot{\tau}(t) \leq \tau<1,0 \leq \mu(t) \leq \mu_{N}, \dot{\mu}(t) \leq \mu<1,
\end{aligned}
$$


where $\tau_{N}, \mu_{N}$ are constants. The initial value associated with (2.1) is assumed to be $x(s)=$ $\psi(s)$, and $\psi(s)$ is a continuous function on $[-h, 0], h=\max \left\{\tau_{N}, \mu_{N}\right\}$.

With loss of generality, it is assumed that the above neural networks have only one equilibrium point and are denoted by $x^{*}=\left(x_{1}^{*}, x_{2}^{*}, \ldots, x_{n}^{*}\right)^{T}$. For the purpose of simplicity, the equilibrium $x^{*}$ will be always shifted to the origin by letting $y(t)=x(t)-x^{*}$, and the neural network system (2.1) can be represented as follows:

$$
\dot{y}(t)=-A y(t)+W_{1} f(y(t))+W_{2} f(y(t-\tau(t)))+W_{3} \int_{t-\tau(t)}^{t} f(y(s)) d s+W_{4} \dot{y}(t-\mu(t)),
$$

where $f_{j}\left(y_{j}(t)\right)=g_{j}\left(y_{j}(t)+x_{j}^{*}\right)-g_{j}\left(x_{j}^{*}\right)$, and $f_{j}(0)=0, j=1,2, \ldots, n$.

The initial condition associated with (2.2) is given in the form $y(s)=x(s)-x^{*}=$ $\varphi(s)=\psi(s)-x^{*}, s \in[-h, 0]$. From the assumption $\left(\mathscr{L}_{1}\right)$, it follows that $f(y(t)) \leq K y(t), K=$ $\operatorname{diag}\left(k_{1}, k_{2}, \ldots, k_{n}\right)$. Based on some transformations, the system (2.2) can be written as an equivalent form

$$
\begin{aligned}
\dot{y}(t)= & -\left[A_{0}+E_{A} \Sigma_{A} F_{A}\right] y(t)+\left[W_{10}+E_{1} \Sigma_{1} F_{1}\right] f(y(t))+\left[W_{20}+E_{2} \Sigma_{2} F_{2}\right] f(y(t-\tau(t))) \\
& +\left[W_{30}+E_{3} \Sigma_{3} F_{3}\right] \int_{t-\tau(t)}^{t} f(y(s)) d s+\left[W_{40}+E_{4} \Sigma_{4} F_{4}\right] \dot{y}(t-\mu(t))
\end{aligned}
$$

where $\Sigma_{A} \in \Sigma, \Sigma_{k} \in \Sigma, k=1,2,3,4$

$$
\begin{gathered}
\Sigma=\left\{\operatorname{diag}\left[\delta_{11}, \ldots, \delta_{1 n}, \ldots, \delta_{n 1}, \ldots, \delta_{n n}\right] \in R^{n^{2} \times n^{2}}:\left|\delta_{i j}\right| \leq 1, i, j=1,2, \ldots, n\right\}, \\
A_{0}=\frac{\bar{A}+\underline{A}}{2}, \quad H_{A}=\left[\alpha_{i j}\right]_{n \times n}=\frac{\bar{A}-\underline{A}}{2}, \\
W_{k 0}=\frac{\bar{W}_{k}+\underline{W_{k}}}{2}, \quad H_{W}^{(k)}=\left[\beta_{i j}\right]_{n \times n}=\frac{\bar{W}_{k}-\underline{W_{k}}}{2}, \\
E_{A}=\left[\sqrt{\alpha_{11}} e_{1}, \ldots, \sqrt{\alpha_{1 n}} e_{1}, \ldots, \sqrt{\alpha_{n 1}} e_{n}, \ldots, \sqrt{\alpha_{n n}} e_{n}\right]_{n \times n^{2}} \\
F_{A}=\left[\sqrt{\alpha_{11}} e_{1}, \ldots, \sqrt{\alpha_{1 n}} e_{n}, \ldots, \sqrt{\alpha_{n 1}} e_{1}, \ldots, \sqrt{\alpha_{n n}} e_{n}\right]_{n^{2} \times n^{\prime}}^{T} \\
E_{k}=\left[\sqrt{\beta_{11}^{(k)}} e_{1}, \ldots, \sqrt{\beta_{1 n}^{(k)}} e_{1}, \ldots, \sqrt{\beta_{n 1}^{(k)}} e_{n}, \ldots, \sqrt{\beta_{n n}^{(k)}} e_{n}\right]_{n \times n^{2}}^{\prime} \\
F_{k}=\left[\sqrt{\beta_{11}^{(k)}} e_{1}, \ldots, \sqrt{\beta_{1 n}^{(k)}} e_{n}, \ldots, \sqrt{\beta_{n 1}^{(k)}} e_{1}, \ldots, \sqrt{\beta_{n n}^{(k)}} e_{n}\right]_{n^{2} \times n}^{T}
\end{gathered}
$$

where $e_{i} \in R^{n}$ denotes the column vector with $i$ th element to be 1 and others to be 0 .

The switched interval neural networks with discrete and distributed time-varying delays of neural type consists of a set of interval neural network with discrete and distributed time-varying delays of neural type and a switching rule. Each of the interval neural networks is regarded as an individual subsystem. The operation mode of the switched neural networks 
is determined by the switching rule. According to (2.2), the switched interval neural network with discrete and distributed delays of neural type can be described as follows:

$$
\begin{aligned}
\dot{y}(t)=- & A_{\sigma(t)} y(t)+W_{1_{\sigma(t)}} f(y(t))+W_{2_{\sigma(t)}} f(y(t-\tau(t)))+W_{3_{\sigma(t)}} \int_{t-\tau(t)}^{t} f(y(s)) d s \\
& +W_{4_{\sigma(t)}} \dot{y}(t-\mu(t)), \quad A_{\sigma(t)} \in A_{l_{\sigma(t)}}, \quad W_{k_{\sigma(t)}} \in W_{l_{\sigma(t)}}^{(k)}, \quad k=1,2,3,4,
\end{aligned}
$$

where $A_{l_{\sigma(t)}}=\left[\underline{A}_{\sigma(t)}, \bar{A}_{\sigma(t)}\right]=\left\{A_{\sigma(t)}=\operatorname{diag}\left(a_{i_{\sigma(t)}}\right): 0<\underline{a}_{i_{\sigma(t)}} \leq a_{i_{\sigma(t)}} \leq \bar{a}_{i_{\sigma(t)}}, i=1,2, \ldots, n\right\}$, $W_{l_{\sigma(t)}}^{(k)}=\left[\underline{W}_{k_{\sigma(t)}}, \bar{W}_{k_{\sigma(t)}}\right]=\left\{W_{k_{\sigma(t)}}=\left[w_{i j_{\sigma(t)}}^{(k)}\right]: 0<\underline{w}_{i j_{\sigma(t)}}^{(k)} \leq w_{i j_{\sigma(t)}}^{(k)} \leq \bar{w}_{i j_{\sigma(t)}}^{(k)}, i, j=1,2, \ldots, n\right\}$ with $\underline{A}_{\sigma(t)}=\operatorname{diag}\left(\underline{a}_{1_{\sigma(t)}} \underline{a}_{2_{\sigma(t)}}, \ldots, \underline{a}_{n_{\sigma(t)}}\right), \bar{A}_{\sigma(t)}=\operatorname{diag}\left(\bar{a}_{1_{\sigma(t)}}, \bar{a}_{2_{\sigma(t)}}, \ldots, \bar{a}_{n_{\sigma(t)}}\right), \underline{W}_{k_{\sigma(t)}}=\left[\underline{w}_{i j_{\sigma(t)}}^{(k)}\right]_{n \times n^{\prime}}$, $\bar{W}_{k_{\sigma(t)}}=\left[\bar{w}_{i j_{\sigma(t)}}^{(k)}\right]_{n \times n}$

$$
\begin{aligned}
& A_{0_{\sigma(t)}}=\frac{\bar{A}_{\sigma(t)}+\underline{A}_{\sigma(t)}}{2}, \quad H_{A_{\sigma(t)}}=\left[\alpha_{i j_{\sigma(t)}}\right]_{n \times n}=\frac{\bar{A}_{\sigma(t)}-\underline{A}_{\sigma(t)}}{2}, \\
& W_{k 0_{\sigma(t)}}=\frac{\bar{W}_{k_{\sigma(t)}}+\underline{W}_{k_{\sigma(t)}}}{2}, \quad H_{W_{\sigma(t)}}^{(k)}=\left[\beta_{i j_{\sigma(t)}}\right]_{n \times n}=\frac{\bar{W}_{k_{\sigma(t)}}-\underline{W}_{k_{\sigma(t)}}}{2} \text {, } \\
& E_{A_{\sigma(t)}}=\left[\sqrt{\alpha_{11_{\sigma(t)}}} e_{1}, \ldots, \sqrt{\alpha_{1 n_{\sigma(t)}}} e_{1}, \ldots, \sqrt{\alpha_{n 1_{\sigma(t)}}} e_{n}, \ldots, \sqrt{\alpha_{n n_{\sigma(t)}}} e_{n}\right]_{n \times n^{2}} \\
& F_{A_{\sigma(t)}}=\left[\sqrt{\alpha_{11_{\sigma(t)}}} e_{1}, \ldots, \sqrt{\alpha_{1 n_{\sigma(t)}}} e_{n}, \ldots, \sqrt{\alpha_{n 1_{\sigma(t)}}} e_{1}, \ldots, \sqrt{\alpha_{n n_{\sigma(t)}}} e_{n}\right]_{n^{2} \times n^{\prime}}^{T}, \\
& E_{k_{\sigma(t)}}=\left[\sqrt{\beta_{11_{\sigma(t)}}^{(k)}} e_{1}, \ldots, \sqrt{\beta_{1 n_{\sigma(t)}^{(k)}}^{(k)}} e_{1}, \ldots, \sqrt{\beta_{n 1_{\sigma(t)}^{(k)}}^{(t)}} e_{n}, \ldots, \sqrt{\beta_{n n_{\sigma(t)}}^{(k)}} e_{n}\right]_{n \times n^{2}}, \\
& F_{k_{\sigma(t)}}=\left[\sqrt{\beta_{11_{\sigma(t)}}^{(k)}} e_{1}, \ldots, \sqrt{\beta_{1 n_{\sigma(t)}}^{(k)}} e_{n}, \ldots, \sqrt{\beta_{n 1_{\sigma(t)}}^{(k)}} e_{1}, \ldots, \sqrt{\beta_{n n_{\sigma(t)}}^{(k)}} e_{n}\right]_{n^{2} \times n}^{T},
\end{aligned}
$$

$\sigma(t):[0,+\infty) \rightarrow \Gamma=\{1,2, \ldots, N\}$ is the switching signal, which is a piecewise constant function of time. For any $i \in\{1,2, \ldots, l\}, A_{i}=A_{0_{i}}+E_{A_{i}} \Sigma_{A_{i}} F_{A_{i}}, W_{k_{i}}=$ $W_{k 0_{i}}+E_{k_{i}} \Sigma_{k_{i}} F_{k_{i}}$, and $\Sigma_{A_{i}} \in \Sigma, \Sigma_{k_{i}} \in \Sigma, k=1,2,3,4$. This means that the matrices $\left(A_{\sigma(t)}, W_{1_{\sigma(t)}}, W_{2_{\sigma(t)}}, W_{3_{\sigma(t)}}, W_{4_{\sigma(t)}}\right)$ are allowed to take values, at an arbitrary time, in the finite set $\left\{\left(A_{1}, W_{1_{1}}, W_{2_{1}}, W_{3_{1}}, W_{4_{1}}\right),\left(A_{2}, W_{1_{2}}, W_{2_{2}}, W_{3_{2}}, W_{4_{2}}\right), \ldots,\left(A_{N}, W_{1_{N}}, W_{2_{N}}, W_{3_{N}}, W_{4_{N}}\right)\right\}$. Throughout this paper, it is assumed that the switching rule $\sigma$ is not known a priori, and its instantaneous value is available in real time. The initial condition associated with the switching system $(2.5)$ is $y(s)=\varphi(s), s \in[-h, 0]$.

By (2.3), the system (2.5) can be written as

$$
\begin{aligned}
\dot{y}(t)= & -A_{0_{\sigma(t)}} y(t)+W_{10_{\sigma(t)}} f(y(t))+W_{20_{\sigma(t)}} f(y(t-\tau(t)))+W_{30_{\sigma(t)}} \int_{t-\tau(t)}^{t} f(y(s)) d s \\
& +W_{40_{\sigma(t)}} \dot{y}(t-\mu(t))+E_{\sigma(t)} \Delta_{\sigma(t)}(t), \quad A_{\sigma(t)} \in A_{l_{\sigma(t)}}, W_{k_{\sigma(t)}} \in W_{l_{\sigma(t)}}^{(k)}, k=1,2,3,4,
\end{aligned}
$$


where $E_{\sigma(t)}=\left[E_{A_{\sigma(t)}}, E_{1_{\sigma(t)}}, E_{2_{\sigma(t)}}, E_{3_{\sigma(t)}}, E_{4_{\sigma(t)}}\right]$

$$
\begin{aligned}
& \Delta_{\sigma(t)}(t)=\left[\begin{array}{c}
-\Sigma_{A_{\sigma(t)}} F_{A_{\sigma(t)}} y(t) \\
\Sigma_{1_{\sigma(t)}} F_{1_{\sigma(t)}} f(y(t)) \\
\Sigma_{2_{\sigma(t)}} F_{2_{\sigma(t)}} f(y(t-\tau(t))) \\
\Sigma_{3_{\sigma(t)} F_{3_{\sigma(t)}}} \int_{t-\tau(t)}^{t} f(y(s)) d s \\
\Sigma_{4_{\sigma(t)}} F_{4_{\sigma(t)}} \dot{y}(t-\mu(t))
\end{array}\right] \\
& =\operatorname{diag}\left\{\Sigma_{A_{\sigma(t)}}, \Sigma_{1_{\sigma(t)}}, \Sigma_{2_{\sigma(t)}}, \Sigma_{3_{\sigma(t)},}, \Sigma_{4_{\sigma(t)}}\right\}\left[\begin{array}{c}
-F_{A_{\sigma(t)}} y(t) \\
F_{1_{\sigma(t)}} f(y(t)) \\
F_{2_{\sigma(t)}} f(y(t-\tau(t))) \\
F_{3_{\sigma(t)}} \int_{t-\tau(t)}^{t} f(y(s)) d s \\
F_{4_{\sigma(t)}} \dot{y}(t-\mu(t))
\end{array}\right],
\end{aligned}
$$

and $\Delta_{\sigma(t)}(t)$ satisfies the following quadratic inequality:

$$
\Delta_{\sigma(t)}^{T}(t) \Delta_{\sigma(t)}(t) \leq\left[\begin{array}{c}
y(t) \\
f(y(t)) \\
f(y(t-\tau(t))) \\
\int_{t-\tau(t)}^{t} f(y(s)) d s \\
\dot{y}(t-\mu(t))
\end{array}\right]^{T}\left[\begin{array}{c}
F_{A_{\sigma(t)}}^{T} \\
F_{1_{\sigma(t)}}^{T} \\
F_{2_{\sigma(t)}}^{T} \\
F_{3_{\sigma(t)}}^{T} \\
F_{4_{\sigma(t)}}^{T}
\end{array}\right]\left[\begin{array}{c}
F_{A_{\sigma(t)}}^{T} \\
F_{1_{\sigma(t)}}^{T} \\
F_{2_{\sigma(t)}^{T}}^{T} \\
F_{3_{\sigma(t)}^{T}}^{T} \\
F_{4_{\sigma(t)}}^{T}
\end{array}\right]^{T}\left[\begin{array}{c}
y(t) \\
f(y(t)) \\
f(y(t-\tau(t))) \\
\int_{t-\tau(t)}^{t} f(y(s)) d s \\
\dot{y}(t-\mu(t))
\end{array}\right] .
$$

Define the indicator function $\xi(t)=\left[\xi_{1}(t), \xi_{2}(t), \ldots, \xi_{N}(t)\right]^{T}$, where

$$
\xi_{i}(t)= \begin{cases}1, & \text { when the switched system is described by the } i \text { th mode } \\ & A_{0_{i}}, W_{k 0_{i}}, k=1,2,3,4, E_{i} \\ 0, & \text { otherwise }\end{cases}
$$

where $i=1,2, \ldots, N$. Therefore, the system model (2.7) can also be written as

$$
\begin{aligned}
\dot{y}(t)=\sum_{i=1}^{N} \xi_{i}(t)\{ & -A_{0_{i}} y(t)+W_{10_{i}} f(y(t))+W_{20_{i}} f(y(t-\tau(t))) \\
& \left.+W_{30_{i}} \int_{t-\tau(t)}^{t} f(y(s)) d s+W_{40_{i}} \dot{y}(t-\mu(t))+E_{i} \Delta_{i}(t)\right\}
\end{aligned}
$$

where $\sum_{i=1}^{N} \xi_{i}(t)=1$ is satisfied under any switching rules. 

be need.

To derive the main result in the next section, the following definitions and lemmas will

Definition 2.1. The switched interval neural network model (2.5) is said to be globally robustly asymptotically stable if the neural network model (2.5) is globally asymptotically stable for any $A_{\sigma(t)} \in A_{l_{\sigma(t)}}, W_{k_{\sigma(t)}} \in W_{l_{\sigma(t)}}^{(k)}, k=1,2,3,4$.

Lemma 2.2 (see [6]). Let $\Gamma_{0}(x)$ and $\Gamma_{1}(x)$ be two arbitrary quadratic forms over $R^{n}$, then $\Gamma_{0}(x)<0$ for all $x \in R^{n}-\{0\}$ satisfying $\Gamma_{1}(x) \leq 0$ if and only if there exists $\varepsilon \geq 0$ such that

$$
\Gamma_{0}(x)-\varepsilon \Gamma_{1}(x)<0, \quad \forall x \in R^{n}-\{0\} .
$$

Lemma 2.3 (Jensen's inequality, see [23]). For any constant matrix $\Omega \in R^{n \times n}, \Omega=\Omega^{T}>$ 0 , scalar $0<\gamma(t)<\gamma$, vector function $\omega:[t-\gamma, t] \rightarrow R^{n}, t \geq 0$ such that the integrations concerned are well defined, then

$$
\left(\int_{0}^{\gamma(t)} \omega(s) d s\right)^{T} \Omega\left(\int_{0}^{\gamma(t)} \omega(s) d s\right) \leq \gamma(t)\left(\int_{0}^{\gamma(t)} \omega(s)^{T} \Omega \omega(s) d s\right) .
$$

\section{Main Results}

In this section, the global robust asymptotic stability of the proposed model (2.5) will be discussed. By constructing a suitable Lyapunov functional, a robust delay-dependent criterion for the global asymptotic stability of the neural network system (2.5) is derived in terms of LMIs.

Theorem 3.1. Under the assumptions $\left(\mathscr{L}_{1}\right)$ and $\left(\mathscr{L}_{2}\right)$, if there exist matrices $P>0, Q_{1}>0, Q_{2}>$ $0, Q_{3}>0, Q_{4}>0, N_{i}(i=1,2, \ldots, 7)$ such that the following LMIs hold:

$$
\Pi_{i}=\left[\begin{array}{ccccccc}
\prod_{i 11} & \prod_{i 12} & \prod_{i 13} & \prod_{i 14} & \prod_{i 15} & \prod_{i 16} & \prod_{i 17} \\
* & -Q_{1} & N_{3} W_{20_{i}} & N_{3} W_{30_{i}} & N_{3} W_{40_{i}} & N_{3} E_{i} & -N_{3} \\
* & * & \prod_{i 33} & \prod_{i 34} & \prod_{i 35} & \prod_{i 36} & N_{2} W_{20_{i}}-N_{4} \\
* & * & * & \prod_{i 44} & \prod_{i 45} & N_{5} E_{i}+N_{7} W_{30_{i}} & N_{2} W_{30_{i}}-N_{5} \\
* & * & * & * & \prod_{i 55} & N_{6} E_{i}+N_{7} W_{40_{i}} & N_{2} W_{40_{i}}-N_{6} \\
* & * & * & * & * & N_{7} E_{i}-I & N_{2} E_{i}-N_{7} \\
* & * & * & * & * & * & Q_{4}-N_{2}-N_{2}^{T}
\end{array}\right]<0,
$$


where

$$
\begin{aligned}
& \prod_{i 11}=-P A_{0_{i}}-A_{0_{i}}^{T} P+P W_{10_{i}} W_{10_{i}}^{T} P+K^{T} K+K Q_{2} K+\tau_{N} K Q_{3} K-N_{1} A_{0_{i}}-A_{0_{i}}^{T} N_{1}^{T}+N_{1} W_{10_{i}} \\
& +W_{10_{i}}^{T} N_{1}^{T}+F_{A_{i}}^{T} F_{A_{i}}+F_{A_{i}}^{T} F_{1_{i}}+F_{1_{i}}^{T} K F_{1_{i}}, \\
& \prod_{i 12}=-N_{3} A_{0_{i}}+N_{3} W_{10_{i}} K, \\
& \prod_{i 13}=-P W_{20_{i}}+N_{1} W_{20_{i}}-N_{4} A_{0_{i}}+N_{4} W_{10_{i}} K+F_{A_{i}}^{T} F_{2_{i}}+F_{1_{i}}^{T} K F_{2_{i}}, \\
& \prod_{i 14}=-P W_{30_{i}}+N_{1} W_{30_{i}}-N_{5} A_{0_{i}}+N_{5} W_{10_{i}} K+F_{A_{i}}^{T} F_{3_{i}}+F_{1_{i}}^{T} K F_{3_{i}}, \\
& \prod_{i 15}=-P W_{40_{i}}+N_{1} W_{40_{i}}-N_{6} A_{0_{i}}+N_{6} W_{10_{i}} K+F_{A_{i}}^{T} F_{4_{i}}+F_{1_{i}}^{T} K F_{4_{i}} \\
& \prod_{i 16}=-P E_{i}+N_{1} E_{i}-N_{7} A_{0_{i}}+N_{7} W_{10_{i}} K, \\
& \prod_{i 17}=-N_{2} A_{0_{i}}+N_{2} W_{10_{i}} K-N_{1} \\
& \prod_{i 33}=-(1-\tau) Q_{2}+N_{4} W_{20_{i}}+F_{2_{i}}^{T} F_{2_{i}}, \\
& \prod_{i 34}=N_{4} W_{30_{i}}+N_{5} W_{20_{i}}+F_{2_{i}}^{T} F_{3_{i}} \\
& \prod_{i 35}=N_{4} W_{40_{i}}+N_{6} W_{20_{i}}+F_{2 i}^{T} F_{4_{i}} \\
& \prod_{i 36}=N_{4} E_{i}+N_{7} W_{20_{i}}, \\
& \prod_{i 44}=-(1-\tau) 1 / \tau_{N} Q_{2}+N_{5} W_{30_{i}}+F_{3_{i}}^{T} F_{3_{i}}, \\
& \prod_{i 45}=N_{5} W_{40_{i}}+N_{6} W_{30_{i}}+F_{3_{i}}^{T} F_{4_{i}} \\
& \prod_{i 55}=-(1-\mu) Q_{4}+N_{6} W_{40_{i}}+F_{4_{i}}^{T} F_{4_{i}},
\end{aligned}
$$


then the switched interval neural network model (2.5) is globally robustly asymptotically stable under any switching rules.

Proof. Consider the following Lyapunov-Krasovskii functional:

$$
\begin{aligned}
V(t)= & y^{T}(t) P y(t)+\int_{t-\tau(t)}^{t} y(s)^{T} Q_{1} y(s) d s+\int_{t-\tau(t)}^{t} f(y(s))^{T} Q_{2} f(y(s)) d s \\
& +\int_{t-\tau(t)}^{t}(s-t+\tau(t)) f(y(s))^{T} Q_{3} f(y(s)) d s+\int_{t-\mu(t)}^{t} \dot{y}(s)^{T} Q_{4} \dot{y}(s) d s .
\end{aligned}
$$

Calculating the time derivative of $V(t)$ along the trajectory of (2.11), it can follow that

$$
\begin{aligned}
\dot{V}(t)= & 2 y^{T}(t) P \dot{y}(t)+y^{T}(t) Q_{1} y(t)-y(t-\tau(t))^{T} Q_{1} y(t-\tau(t))+f(y(t))^{T} Q_{2} f(y(t)) \\
& -(1-\dot{\tau}(t)) f^{T}(y(t-\tau(t))) Q_{2} f(y(t-\tau(t)))+\tau(t) f^{T}(y(t)) Q_{3} f(y(t)) \\
& +\int_{t-\tau(t)}^{t}(-1+\dot{\tau}(t)) f(y(s))^{T} Q_{3} f(y(s)) d s+\dot{y}^{T}(t) Q_{4} \dot{y}(t) \\
& -\dot{y}^{T}(t-\mu(t)) Q_{4} \dot{y}(t-\mu(t))(1-\dot{\mu}(t)) \\
= & 2 y^{T}(t) P\left\{\sum _ { i = 1 } ^ { N } \xi _ { i } ( t ) \left[-A_{0 i} y(t)+W_{10 i} f(y(t))+W_{20 i} f(y(t-\tau(t)))\right.\right. \\
& \left.\left.\quad+W_{30 i} \int_{t-\tau(t)}^{t} f(y(s)) d s+W_{40 i} \dot{y}(t-\mu(t))+E_{i} \Delta_{i}(t)\right]\right\} \\
& +y^{T}(t) Q_{1} y(t)-y(t-\tau(t))^{T} Q_{1} y(t-\tau(t))+f(y(t))^{T} Q_{2} f(y(t)) \\
& -(1-\dot{\tau}(t)) f^{T}(y(t-\tau(t))) Q_{2} f(y(t-\tau(t)))+\tau(t) f^{T}(y(t)) Q_{3} f(y(t)) \\
& +\int_{t-\tau(t)}^{t}(-1+\dot{\tau}(t)) f(y(s))^{T} Q_{3} f(y(s)) d s+\dot{y}^{T}(t) Q_{4} \dot{y}(t) \\
& -\dot{y}^{T}(t-\mu(t)) Q_{4} \dot{y}(t-\mu(t))(1-\dot{\mu}(t)) \\
= & +\sum_{i=1}^{N} \xi_{i}(t)\left\{y^{T}(t)\left(-P A_{0 i}-A_{0 i}^{T} P\right) y(t)+2 y^{T}(t) P W_{10 i} f(y(t))\right. \\
& +2 y^{T}(t) P W_{20 i} f(y(t-\tau(t))) \\
& +2 y^{T}(t) P W_{30 i} \int_{t-\tau(t)}^{t} f(y(s)) d s+2 y^{T}(t) P W_{40 i} \dot{y}(t-\mu(t))+2 y^{T}(t) P E_{i} \Delta_{i}(t) \\
& \quad-y(t)-y(t-\tau(t))^{T} Q_{1} y(t-\tau(t))+f(y(t))^{T} Q_{2} f(y(t)) \\
& \\
& \\
& \\
& \\
&
\end{aligned}
$$




$$
\begin{aligned}
& -(1-\dot{\tau}(t)) f^{T}(y(t-\tau(t))) Q_{2} f(y(t-\tau(t)))+\tau(t) f^{T}(y(t)) Q_{3} f(y(t)) \\
& +\int_{t-\tau(t)}^{t}(-1+\dot{\tau}(t)) f(y(s))^{T} Q_{3} f(y(s)) d s+\dot{y}^{T}(t) Q_{4} \dot{y}(t) \\
& \left.-\dot{y}^{T}(t-\mu(t)) Q_{4} \dot{y}(t-\mu(t))(1-\dot{\mu}(t))\right\} .
\end{aligned}
$$

By the assumption $\left(\mathscr{\ell}_{1}\right)$ and Lemma 2.3,

$$
\begin{gathered}
2 y^{T}(t) P W_{10_{i}} f(y(t)) \leq y^{T}(t)\left(P W_{10_{i}} W_{10_{i}}^{T} P+K^{T} K\right) y(t), \\
f(y(t))^{T} Q_{2} f(y(t)) \leq y^{T}(t) K Q_{2} K y(t), \\
\int_{t-\tau(t)}^{t}(-1+\dot{\tau}(t)) f(y(s))^{T} Q_{3} f(y(s)) d s \\
\leq-(1-\tau) \frac{1}{\tau_{N}}\left(\int_{t-\tau(t)}^{t} f(y(s)) d s\right)^{T} Q_{3}\left(\int_{t-\tau(t)}^{t} f(y(s)) d s\right) .
\end{gathered}
$$

Noting the following zero equation with free weighting matrices $N_{i}(i=1,2, \ldots, 7)$, which indicate the relationship between the terms in the state equation (2.11) and can easily be determined by solving the corresponding LMIs, it follows that

$$
\begin{aligned}
2 \zeta(t)^{T} N \times\left\{\sum_{i=1}^{l} \xi_{i}(t)[\right. & -\dot{y}(t)-A_{0_{i}} y(t)+W_{10_{i}} f(y(t))+W_{20_{i}} f(y(t-\tau(t))) \\
& \left.\left.+W_{30_{i}} \int_{t-\tau(t)}^{t} f(y(s)) d s+W_{40_{i}} \dot{y}(t-\mu(t))+E_{i} \Delta_{i}(t)\right]\right\}=0
\end{aligned}
$$

$\zeta(t)=\left[y^{T}(t) \dot{y}^{T}(t) y^{T}(t-\tau(t)) f^{T}(y(t-\tau(t)))\left(\int_{t-\tau(t)}^{t} f(y(s)) d s\right)^{T} \dot{y}^{T}(t-\mu(t)) \Delta_{i}^{T}(t)\right]^{T} \cdot N=$ $\left[N_{1}^{T} N_{2}^{T} N_{3}^{T} N_{4}^{T} N_{5}^{T} N_{6}^{T} N_{7}^{T}\right]^{T}$. By substituting (2.9) and (3.5)-(3.8) into (3.4), it follows that

$$
\begin{aligned}
& \varepsilon \dot{V}(t)-\varepsilon \sum_{i=1}^{N} \xi_{i}(t)\left(\Delta_{i}^{T}(t) \Delta_{i}(t)-\left[\begin{array}{c}
y(t) \\
f(y(t)) \\
f(y(t-\tau(t))) \\
\int_{t-\tau(t)}^{t} f(y(s)) d s \\
\dot{y}(t-\mu(t))
\end{array}\right]^{T}\left[\begin{array}{c}
F_{A_{i}}^{T} \\
F_{1_{i}}^{T} \\
F_{2_{i}}^{T} \\
F_{3_{i}}^{T} \\
F_{4_{i}}^{T}
\end{array}\right]\left[\begin{array}{c}
F_{A_{i}}^{T} \\
F_{1_{i}}^{T} \\
F_{2_{i}}^{T} \\
F_{3_{i}}^{T} \\
F_{4_{i}}^{T}
\end{array}\right]^{T}\left[\begin{array}{c}
y(t) \\
f(y(t)) \\
f(y(t-\tau(t))) \\
\int_{t-\tau(t)}^{t} f(y(s)) d s \\
\dot{y}(t-\mu(t))
\end{array}\right]\right) \\
& \leq \varepsilon \sum_{i=1}^{N} \xi_{i}(t) \phi^{T}(t) \Pi_{i} \phi(t),
\end{aligned}
$$


where $\phi(t)=\left[\begin{array}{ll}y^{T}(t) & y^{T}(t-\tau(t)) f^{T}(y(t-\tau(t)))\left(\int_{t-\tau(t)}^{t} f(y(s)) d s\right)^{T} \dot{y}^{T}(t-\mu(t)) \Delta_{i}^{T}(t) \dot{y}^{T}(t)\end{array}\right]^{T}$ and $\varepsilon>0$. From Lemma 2.2 with the conditions (3.1) and (3.9), this implies that $\dot{V}(t)<0$ for all $\phi(t) \neq 0$. Hence, the neural network system (2.7) is globally asymptotically stable for $A_{\sigma(t)} \in A_{l_{\sigma(t)}}, W_{k_{\sigma(t)}} \in W_{l_{\sigma(t)}}^{(k)}, k=1,2,3,4$, that is, the switched neural network model (2.5) is globally robustly asymptotically stable. The proof is completed.

When the time-varying delay $\tau(t)$ and $\mu(t)$ in (2.5) becomes into $\tau(t)=\tau=$ const, $\mu(t)=\mu=$ const, according to Theorem 3.1, it is easy to obtain the following corollary.

Corollary 3.2. Under the assumption $\left(\mathscr{L}_{1}\right)$ and $\left(\mathscr{\ell}_{2}\right)$, if there exist matrices $P>0, Q_{1}>0, Q_{2}>$ $0, Q_{3}>0, Q_{4}>0, N_{i}(i=1,2, \ldots, 7)$ such that the following LMIs hold:

$$
\left[\begin{array}{ccccccc}
\prod_{i 11} & \prod_{i 12} & \prod_{i 13} & \prod_{i 14} & \prod_{i 15} & \prod_{i 16} & \prod_{i 17} \\
* & -Q_{1} & N_{3} W_{20_{i}} & N_{3} W_{30_{i}} & N_{3} W_{40_{i}} & N_{3} E_{i} & -N_{3} \\
* & * & \prod_{i 33} & \prod_{i 34} & \prod_{i 35} & \prod_{i 36} & N_{2} W_{20_{i}}-N_{4} \\
* & * & * & \prod_{i 44} & \prod_{i 45} & N_{5} E_{i}+N_{7} W_{30_{i}} & N_{2} W_{30_{i}}-N_{5} \\
* & * & * & * & \prod_{i 55} & N_{6} E_{i}+N_{7} W_{40_{i}} & N_{2} W_{40_{i}}-N_{6} \\
* & * & * & * & * & N_{7} E_{i}-I & N_{2} E_{i}-N_{7} \\
* & * & * & * & * & * & Q_{4}-N_{2}-N_{2}^{T}
\end{array}\right]<0,
$$

where

$$
\begin{aligned}
\prod_{i 11}= & -P A_{0_{i}}-A_{0_{i}}^{T} P+P W_{10_{i}} W_{10_{i}}^{T} P+K^{T} K+K Q_{2} K+\tau_{N} K Q_{3} K-N_{1} A_{0_{i}}-A_{0_{i}}^{T} N_{1}^{T} \\
& +N_{1} W_{10_{i}}+W_{10_{i}}^{T} N_{1}^{T}+F_{A_{i}}^{T} F_{A_{i}}+F_{A_{i}}^{T} F_{1_{i}}+F_{1_{i}}^{T} K F_{1_{i}}, \\
\prod_{i 12}= & -N_{3} A_{0_{i}}+N_{3} W_{10_{i}} K \\
\prod_{i 13}= & -P W_{20_{i}}+N_{1} W_{20_{i}}-N_{4} A_{0_{i}}+N_{4} W_{10_{i}} K+F_{A_{i}}^{T} F_{2_{i}}+F_{1_{i}}^{T} K F_{2_{i}}, \\
\prod_{i 14}= & -P W_{30_{i}}+N_{1} W_{30_{i}}-N_{5} A_{0_{i}}+N_{5} W_{10_{i}} K+F_{A_{i}}^{T} F_{3_{i}}+F_{1_{i}}^{T} K F_{3_{i}}, \\
\prod_{i 15}= & -P W_{40_{i}}+N_{1} W_{40_{i}}-N_{6} A_{0_{i}}+N_{6} W_{10_{i}} K+F_{A_{i}}^{T} F_{4_{i}}+F_{1_{i}}^{T} K F_{4_{i}}, \\
\prod_{i 16}= & -P E_{i}+N_{1} E_{i}-N_{7} A_{0_{i}}+N_{7} W_{10_{i}} K, \\
\prod_{i 17}= & -N_{2} A_{0_{i}}+N_{2} W_{10_{i}} K-N_{1},
\end{aligned}
$$




$$
\begin{aligned}
& \prod_{i 33}=-Q_{2}+N_{4} W_{20_{i}}+F_{2_{i}}^{T} F_{2_{i},} \\
& \prod_{i 34}=N_{4} W_{30_{i}}+N_{5} W_{20_{i}}+F_{2_{i}}^{T} F_{3_{i},} \\
& \prod_{i 35}=N_{4} W_{40_{i}}+N_{6} W_{20_{i}}+F_{2_{i}}^{T} F_{4_{i}}, \\
& \prod_{i 36}=N_{4} E_{i}+N_{7} W_{20_{i},} \\
& \prod_{i 44}=-1 / \tau Q_{2}+N_{5} W_{30_{i}}+F_{3_{i}}^{T} F_{3_{i}}, \\
& \prod_{i 45}=N_{5} W_{40_{i}}+N_{6} W_{30_{i}}+F_{3_{i}}^{T} F_{4_{i}}, \\
& \prod_{i 55}=-Q_{4}+N_{6} W_{40_{i}}+F_{4_{i}}^{T} F_{4_{i}},
\end{aligned}
$$

then the switched interval neural network model (2.5) is global robust asymptotically stable.

Remark 3.3. When $N=1$, the switched system model (2.7) degenerated into the interval neural network model (2.1) with discrete and distributed time-varying delays of neutral type which contain neural network models studied in $[1-3,5,7]$. Moreover, note that when $N=$ 1 , $W_{1}=0$, and $\tau(t)=\mu(t)=$ const, Theorem 3.1 in this paper coincides with Theorem 1 in [12]. Without considering interval parameter uncertainty, the neural network models presented in [11,13-15] are the special case. Hence, the results obtained in this paper extend and improve the stability results available in the existing literature $[1-3,5,7,11,13-15]$.

Remark 3.4. In this paper, augmented Lyapunov functional is used to analyze the stability of the interval neural network model (2.1) with discrete and distributed time-varying delays of neutral type. In the Lyapunov functional, both state and activation function are considered in the same term. Hence, the novel Lyapunov function contains structures more general than the traditional ones, and the negative matrices $\Pi_{i}$ in Theorem 3.1 contain more elements. This shows that it is easy to find more appropriate elements in $\Pi_{i}$ to ensure that the LMIs (3.1) hold. Thus, sufficient conditions given in this paper are less conservative than the existing results.

Remark 3.5. In this paper, the activation function is Lipschitz continuous, which is first introduced in [29] and used also in [30,31] is more general than the usual sigmoid functions. Therefore, the stability results obtained in this paper are less conservative than those in [1013, 27].

Remark 3.6. In $[32,33]$, the mixed time-delay problems have been considered for the stochastic system with Markovian jump parameters and discrete-time stochastic complex networks with randomly occurred nonlinearities. By applying the Lyapunov-Krasovskii functional approach and linear matrix inequality techniques, the conditions of exponential 
stabilization and global synchronization were presented for the stochastic system and discrete-time stochastic complex networks. In [34], Bounded H-infinity synchronization and state estimation were considered for discrete time-varying stochastic complex networks over a finite- horizon via linear matrix inequality. [35] The authors studied the robust H-infinity fuzzy output-feedback control; a controller design is given for the system with multiple probabilistic delays and multiple missing measurements. In the future, based on [32, 33], the model of the switched interval stochastic system and the switched interval discrete-time stochastic complex networks will be expected to be established, and the stability strategy proposed in this paper will be utilized to investigate the stability problems.

\section{An Illustrative Example}

In this section, an example will be given to illustrate the validity and effectiveness of the proposed stability criterion for the switched interval neural network with discrete and distributed delays of neural type when $N=2$.

Example 4.1. Consider the following second-order switched interval neural networks with discrete and distributed delays of neural type:

$$
\begin{gathered}
\dot{y}_{i}(t)=-a_{i_{\sigma(t)}} y_{i}(t)+\sum_{j=1}^{2} w_{i j_{\sigma(t)}}^{(1)} f_{j}\left(y_{j}(t)\right)+\sum_{j=1}^{2} w_{i j_{\sigma(t)}}^{(2)} f_{j}\left(y_{j}(t-\tau(t))\right)+\sum_{j=1}^{2} w_{i j_{\sigma(t)}}^{(3)} \int_{t-\tau(t)}^{t} f_{j}\left(y_{j}(s)\right) d s \\
+\sum_{j=1}^{2} w_{i j_{\sigma(t)}}^{(4)} \dot{y}_{j}(t-\mu(t)), \quad a_{i_{\sigma(t)}} \in\left[\underline{a}_{i_{\sigma(t)}}, \bar{a}_{i_{\sigma(t)}}\right], \quad w_{i j_{\sigma(t)}}^{(k)} \in\left[\underline{w}_{i j_{\sigma(t)}}^{(k)}, \bar{w}_{i j_{\sigma(t)}}^{(k)}\right], \\
k=1,2,3,4, \quad y_{i}(t)=\varphi_{i}(t), \quad t \in[-h, 0], \quad i, j=1,2,
\end{gathered}
$$

where the switching signal $\sigma(t):[0,+\infty) \rightarrow \Gamma=\{1,2\}$, the activation functions $f_{i}(x)=$ $(2 / 3) \sin x+(1 / 3) x, i=1,2$, the discrete and distributed delays $\tau(t)=(1 / 2) \cos t+(1 / 2)$, and the neural type delay $\mu(t)=(1 / 2) \sin t+(1 / 2)$. Obviously, the assumptions $\mathscr{d}_{1}$ and $\mathscr{L}_{2}$ are satisfied with $K=\operatorname{diag}(1,1)$ and $h=1, \tau=\mu=1 / 2$. The neural network system parameters are defined as

$$
\begin{aligned}
& \underline{A}_{1}=\left(\begin{array}{cc}
3.99 & 0 \\
0 & 2.99
\end{array}\right), \quad \bar{A}_{1}=\left(\begin{array}{cc}
4.01 & 0 \\
0 & 3.01
\end{array}\right), \\
& \underline{W}_{11}=\left(\begin{array}{cc}
1.188 & 0.09 \\
0.09 & 1.188
\end{array}\right), \quad \bar{W}_{11}=\left(\begin{array}{cc}
1.208 & 0.11 \\
0.11 & 1.208
\end{array}\right), \\
& \underline{W}_{21}=\left(\begin{array}{cc}
0.09 & 0.14 \\
0.05 & 0.09
\end{array}\right), \quad \bar{W}_{21}=\left(\begin{array}{ll}
0.11 & 0.16 \\
0.07 & 0.11
\end{array}\right), \\
& \underline{W}_{31}=\left(\begin{array}{cc}
0.44 & -0.21 \\
0.29 & 0.41
\end{array}\right), \quad \bar{W}_{31}=\left(\begin{array}{cc}
0.46 & -0.19 \\
0.31 & 0.43
\end{array}\right),
\end{aligned}
$$




$$
\begin{aligned}
& \underline{W}_{41}=\left(\begin{array}{cc}
0.09 & -0.01 \\
-0.01 & 0.09
\end{array}\right), \quad \bar{W}_{41}=\left(\begin{array}{cc}
0.11 & 0.11 \\
0.11 & 0.11
\end{array}\right), \\
& \underline{A}_{2}=\left(\begin{array}{cc}
1.99 & 0 \\
0 & 2.99
\end{array}\right), \quad \bar{A}_{2}=\left(\begin{array}{cc}
2.01 & 0 \\
0 & 3.01
\end{array}\right) \text {, } \\
& \underline{W}_{12}=\left(\begin{array}{ll}
-0.07 & 0.03 \\
-0.01 & 0.02
\end{array}\right), \quad \bar{W}_{12}=\left(\begin{array}{ll}
-0.05 & 0.05 \\
-0.04 & 0.04
\end{array}\right) \text {, } \\
& \underline{W}_{22}=\left(\begin{array}{cc}
-0.47 & -0.15 \\
0.11 & -0.54
\end{array}\right), \quad \bar{W}_{22}=\left(\begin{array}{cc}
-0.45 & -0.13 \\
0.13 & -0.54
\end{array}\right) \text {, } \\
& \underline{W}_{32}=\left(\begin{array}{cc}
-0.31 & 0.09 \\
-0.51 & -0.61
\end{array}\right), \quad \bar{W}_{32}=\left(\begin{array}{cc}
-0.29 & 0.11 \\
-0.49 & -0.59
\end{array}\right) \text {, } \\
& \underline{W}_{42}=\left(\begin{array}{cc}
0.03 & -0.22 \\
-0.21 & -0.44
\end{array}\right), \quad \bar{W}_{42}=\left(\begin{array}{cc}
0.05 & -0.2 \\
-0.19 & -0.42
\end{array}\right) \text {. }
\end{aligned}
$$

Solving the LMI in (3.4) by using appropriate LMI solver in the Matlab, the feasible positive definite matrices $P, Q_{i}, i=1,2,3,4$ and the matrices $N_{i}, i=1,2, \ldots, 7$ could be as

$$
\begin{aligned}
& P=\left(\begin{array}{cc}
6.0192 & -0.5039 \\
-0.5039 & 4.8686
\end{array}\right), \quad Q_{1}=\left(\begin{array}{cc}
3.2386 & 0 \\
0 & 3.2386
\end{array}\right), \quad Q_{2}=\left(\begin{array}{ll}
4.7009 & 0.3313 \\
0.3313 & 4.9146
\end{array}\right), \\
& Q_{3}=\left(\begin{array}{cc}
3.0347 & -0.0788 \\
-0.0788 & 2.8395
\end{array}\right), \quad Q_{4}=\left(\begin{array}{cc}
1.9859 & -0.2388 \\
-0.2388 & 1.4177
\end{array}\right), \quad N_{1}=\left(\begin{array}{cc}
-4.1198 & 0.4992 \\
0.7261 & -2.7597
\end{array}\right), \\
& N_{2}=\left(\begin{array}{cc}
1.9399 & -0.0997 \\
-0.1962 & 1.5020
\end{array}\right), \quad N_{3}=\left(\begin{array}{ll}
0 & 0 \\
0 & 0
\end{array}\right), \quad N_{4}=\left(\begin{array}{cc}
-0.2957 & -0.0263 \\
0.0959 & -0.0107
\end{array}\right) \text {, } \\
& N_{5}=\left(\begin{array}{cc}
-0.0147 & 0.0606 \\
0.1252 & 0.2426
\end{array}\right), \quad N_{6}=\left(\begin{array}{cc}
0.0734 & -0.1495 \\
-0.0498 & -0.0592
\end{array}\right), \quad N_{7}=\left(\begin{array}{ll}
M 1 & M 2
\end{array}\right), \\
& M 1=\left(\begin{array}{cccccccc}
0.085 & 0.085 & -0.0068 & -0.0068 & 0.085 & 0.085 & -0.0068 & -0.0068 \\
0.008 & 0.008 & 0.0892 & 0.0892 & 0.008 & 0.008 & 0.0892 & 0.0892
\end{array}\right) \text {, } \\
& M 2=\left(\begin{array}{cccccccc}
0.085 & 0.085 & -0.0068 & -0.0068 & 0.085 & 0.085 & -0.0068 & -0.0068 \\
0.008 & 0.008 & 0.0892 & 0.0892 & 0.008 & 0.008 & 0.0892 & 0.0892
\end{array}\right) .
\end{aligned}
$$

By Theorem 3.1, this switched interval neural network with discrete and distributed delays of neural type is globally robustly asymptotically stable under any switching rules. 


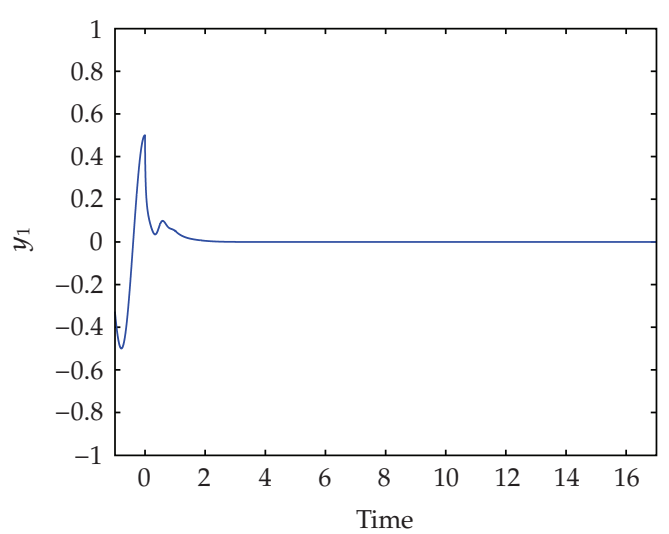

(a)

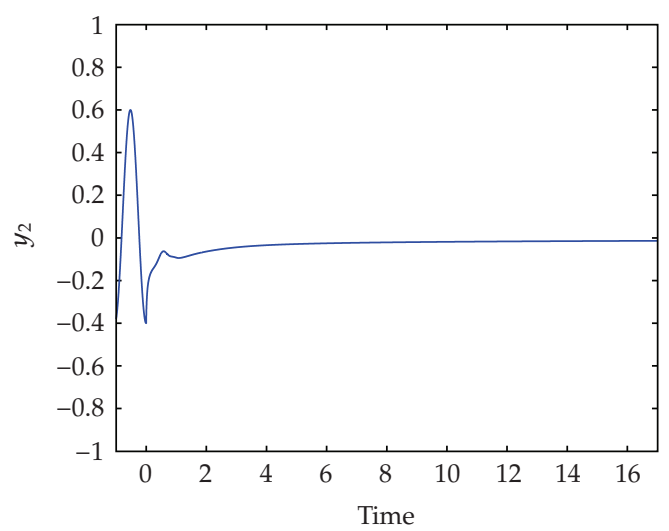

(b)

Figure 1: The state trajectories $y_{1}$ and $y_{2}$ of the network with initial value $\left(\varphi_{1}(t), \varphi_{2}(t)\right)^{T}=$ $\left((\cos 2 t)^{2}-0.5,(\sin 3 t)^{2}-0.4\right)^{T}, t \in[-1,0]$.

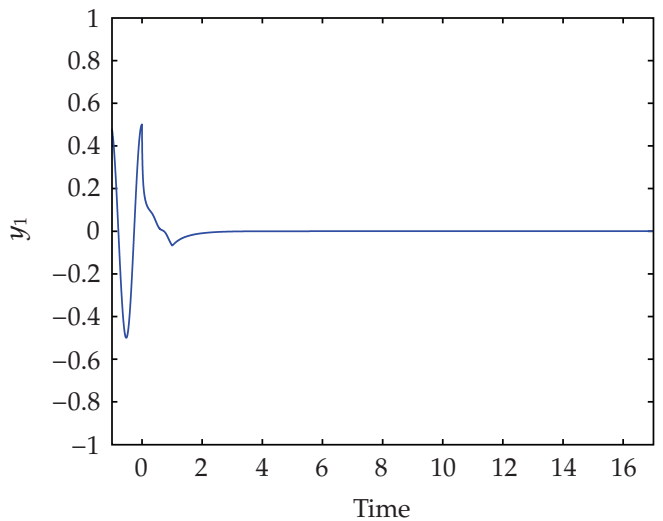

(a)

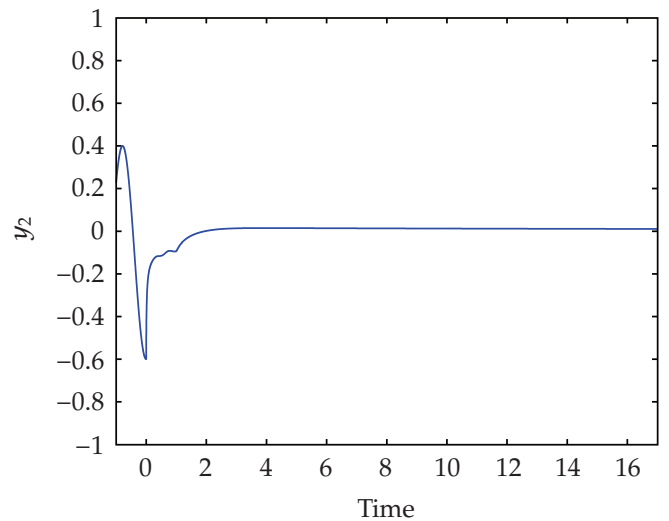

(b)

Figure 2: The state trajectories $y_{1}$ and $y_{2}$ of the network with initial value $\left(\varphi_{1}(t), \varphi_{2}(t)\right)^{T}=$ $\left((\cos 3 t)^{2}-0.5,(\sin 2 t)^{2}-0.6\right)^{T}, t \in[-1,0]$.

Let $A_{1}=\underline{A}_{1}, W_{11}=\underline{W}_{11}, W_{21}=\underline{W}_{21}, W_{31}=\underline{W}_{31}, W_{41}=\underline{W}_{41}$ and $A_{2}=\underline{A}_{2}, W_{12}=$ $\underline{W}_{12}, W_{22}=\underline{W}_{22}, W_{32}=\underline{W}_{32}$, and $W_{42}=\underline{W}_{42}$. For numerical simulation, assume that the two subsystems are switched every four seconds. Figure 1 displays the state trajectories of this network with initial value $\left(\varphi_{1}(t), \varphi_{2}(t)\right)^{T}=\left((\cos 2 t)^{2}-0.5,(\sin 3 t)^{2}-0.4\right)^{T}, t \in[-1,0]$. It can be seen that these trajectories asymptotically converge to the unique equilibrium $x^{*}=(0,0)^{T}$ of the network. This is in accordance with the conclusion of Theorem 3.1.

Let $A_{1}=\bar{A}_{1}, W_{11}=\bar{W}_{11}, W_{21}=\bar{W}_{21}, W_{31}=\bar{W}_{31}, W_{41}=\bar{W}_{41}$ and $A_{2}=\bar{A}_{2}, W_{12}=$ $\bar{W}_{12}, W_{22}=\bar{W}_{22}, W_{32}=\bar{W}_{32}$, and $W_{42}=\bar{W}_{42}$. For numerical simulation, assume that the two subsystems are switched every four seconds. Figure 2 displays the state trajectories of this network with initial value $\left(\varphi_{1}(t), \varphi_{2}(t)\right)^{T}=\left((\cos 3 t)^{2}-0.5,(\sin 2 t)^{2}-0.6\right)^{T}, t \in[-1,0]$. It can be seen that these trajectories asymptotically converge to the unique equilibrium $x^{*}=(0,0)^{T}$ of the network. This is in accordance with the conclusion of Theorem 3.1. 


\section{Conclusion}

In this paper, a novel class of switched interval neural networks with discrete and distributed delays of neural type has been presented by combing the theories of the switched systems and the interval neural networks with discrete and distributed delays of neural type. Furthermore, a delay-dependent criterion expressed in the form of LMIs has been obtained to guarantee the proposed neural networks to be globally asymptotically robustly stable under interval parameter uncertainties. An illustrative example has been also given to demonstrate the effectiveness of the proposed LMI-based stability criteria.

\section{Acknowledgments}

The authors are extremely grateful to the Editor and the Reviewers for their valuable comments and suggestions, which help to enrich the content and improve the presentation of this paper. This work was supported by the Natural Science Foundation of Hebei Province of China (A2011203103) and the Hebei Province Education Foundation of China (2009157).

\section{References}

[1] V. Singh, "A new criterion for global robust stability of interval delayed neural networks," Journal of Computational and Applied Mathematics, vol. 221, no. 1, pp. 219-225, 2008.

[2] V. Singh, "On global robust stability of interval Hopfield neural networks with delay," Chaos, Solitons E Fractals, vol. 33, no. 4, pp. 1183-1188, 2007.

[3] Q. Song and J. Cao, "Global robust stability of interval neural networks with multiple time-varying delays," Mathematics and Computers in Simulation, vol. 74, no. 1, pp. 38-46, 2007.

[4] Y. Q. Zhang and X. Wan, "Statistical fuzzy interval neural networks for currency exchange rate time series prediction," Applied Soft Computing Journal, vol. 7, no. 4, pp. 1149-1156, 2007.

[5] H. Zhang, Z. Wang, and D. Liu, "Robust stability analysis for interval Cohen-Grossberg neural networks with unknown time-varying delays," IEEE Transactions on Neural Networks, vol. 19, no. 11, pp. 1942-1955, 2008.

[6] W. Su and Y. Chen, "Global robust exponential stability analysis for stochastic interval neural networks with time-varying delays," Communications in Nonlinear Science and Numerical Simulation, vol. 14, no. 5, pp. 2293-2300, 2009.

[7] R. Zhang and L. Wang, "Global exponential robust stability of interval cellular neural networks with S-type distributed delays," Mathematical and Computer Modelling, vol. 50, no. 3-4, pp. 380-385, 2009.

[8] H. Wu, F. Tao, L. Qin, R. Shi, and L. He, "Robust exponential stability for interval neural networks with delays and non-Lipschitz activation functions," Nonlinear Dynamics. In press.

[9] S. Senan and S. Arik, "New results for global robust stability of bidirectional associative memory neural networks with multiple time delays," Chaos, Solitons E Fractals, vol. 41, no. 4, pp. 2106-2114, 2009.

[10] R. Rakkiyappan and P. Balasubramaniam, "New global exponential stability results for neutral type neural networks with distributed time delays," Neurocomputing, vol. 71, no. 4-6, pp. 1039-1045, 2008.

[11] R. Rakkiyappan and P. Balasubramaniam, "LMI conditions for global asymptotic stability results for neutral-type neural networks with distributed time delays," Applied Mathematics and Computation, vol. 204, no. 1, pp. 317-324, 2008.

[12] L. Liu, Z. Han, and W. Li, "Global stability analysis of interval neural networks with discrete and distributed delays of neutral type," Expert Systems with Applications, vol. 36, no. 3, pp. 7328-7331, 2009.

[13] J. Zhu, Q. Zhang, and C. Yang, “Delay-dependent robust stability for Hopfield neural networks of neutral-type," Neurocomputing, vol. 72, no. 10-12, pp. 2609-2617, 2009.

[14] J. Qiu and J. Cao, "Delay-dependent robust stability of neutral-type neural networks with time delays," Journal of Mathematical Control Science and Applications, vol. 1, pp. 179-188, 2007. 
[15] J. H. Park, O. M. Kwon, and S. M. Lee, "LMI optimization approach on stability for delayed neural networks of neutral-type," Applied Mathematics and Computation, vol. 196, no. 1, pp. 236-244, 2008.

[16] O. M. Kwon, J. H. Park, and S. M. Lee, "On stability criteria for uncertain delay-differential systems of neutral type with time-varying delays," Applied Mathematics and Computation, vol. 197, no. 2, pp. 864-873, 2008.

[17] J. H. Park, C. H. Park, O. M. Kwon, and S. M. Lee, “A new stability criterion for bidirectional associative memory neural networks of neutral-type," Applied Mathematics and Computation, vol. 199, no. 2, pp. 716-722, 2008.

[18] J. H. Park and O. M. Kwon, "Design of state estimator for neural networks of neutral-type," Applied Mathematics and Computation, vol. 202, no. 1, pp. 360-369, 2008.

[19] Y. Tsividis, "Switched neural networks," US patent no. 4873661, 1989.

[20] T. X. Brown, "Neural networks for switching," IEEE Communications Magazine, vol. 27, no. 11, pp. 72-81, 1989.

[21] M. Muselli, "Gene selection through switched neural networks," in Network Tools and Applications in Biology Workshop (NETTAB '03), pp. 27-28, Bologna, Italy, 2003.

[22] H. Huang, Y. Qu, and H. X. Li, "Robust stability analysis of switched Hopfield neural networks with time-varying delay under uncertainty," Physics Letters A, vol. 345, no. 4-6, pp. 345-354, 2005.

[23] K. Yuan, J. Cao, and H. X. Li, "Robust stability of switched Cohen-Grossberg neural networks with mixed time-varying delays," IEEE Transactions on Systems, Man, and Cybernetics, Part B, vol. 36, no. 6, pp. 1356-1363, 2006.

[24] P. Li and J. Cao, "Global stability in switched recurrent neural networks with time-varying delay via nonlinear measure," Nonlinear Dynamics, vol. 49, no. 1-2, pp. 295-305, 2007.

[25] X. Lou and B. Cui, "Delay-dependent criteria for global robust periodicity of uncertain switched recurrent neural networks with time-varying delay," IEEE Transactions on Neural Networks, vol. 19, no. 4, pp. 549-557, 2008.

[26] J. Lian and K. Zhang, "Exponential stability for switched Cohen-Grossberg neural networks with average dwell time," Nonlinear Dynamics, vol. 63, no. 3, pp. 331-343, 2011.

[27] C. K. Ahn, "Switched exponential state estimation of neural networks based on passivity theory," Nonlinear Dynamics. In press.

[28] S. Boyd, L. El Ghaoui, E. Feron, and V. Balakrishnan, Linear Matrix Inequalities in System and Control Theory, vol. 15 of SIAM Studies in Applied Mathematics, SIAM, Philadelphia, Pa, USA, 1994.

[29] Z. Wang, Y. Liu, and X. Liu, "On global asymptotic stability of neural networks with discrete and distributed delays," Physics Letters A, vol. 345, no. 4-6, pp. 299-308, 2005.

[30] Z. Wang, Y. Liu, M. Li, and X. Liu, "Stability analysis for stochastic Cohen-Grossberg neural networks with mixed time delays," IEEE Transactions on Neural Networks, vol. 17, no. 3, pp. 814-820, 2006.

[31] Z. Wang, D. W.C. Ho, and X. Liu, "State estimation for delayed neural networks," IEEE Transactions on Neural Networks, vol. 16, no. 1, pp. 279-284, 2005.

[32] Z. Wang, Y. Liu, and X. Liu, "Exponential stabilization of a class of stochastic system with Markovian jump parameters and mode-dependent mixed time-delays," IEEE Transactions on Automatic Control, vol. 55, no. 7, pp. 1656-1662, 2010.

[33] Z. Wang, Y. Wang, and Y. Liu, “Global synchronization for discrete-time stochastic complex networks with randomly occurred nonlinearities and mixed time delays," IEEE Transactions on Neural Networks, vol. 21, no. 1, pp. 11-25, 2010.

[34] B. Shen, Z. Wang, and X. Liu, "Bounded $H_{\infty}$ synchronization and state estimation for discrete timevarying stochastic complex networks over a finite-horizon," IEEE Transactions on Neural Networks, vol. 22, pp. 145-157, 2011.

[35] H. Dong, Z. Wang, D. W. C. Ho, and H. Gao, “Robust $H_{\infty}$ fuzzy output-feedback control with multiple probabilistic delays and multiple missing measurements," IEEE Transactions on Fuzzy Systems, vol. 18, no. 4, pp. 712-725, 2010.

[36] S. Xu, J. Lam, D. W. C. Ho, and Y. Zou, “Delay-dependent exponential stability for a class of neural networks with time delays," Journal of Computational and Applied Mathematics, vol. 183, no. 1, pp. 16-28, 2005.

[37] J. Cao, K. Yuan, and H. X. Li, “Global asymptotical stability of recurrent neural networks with multiple discrete delays and distributed delays," IEEE Transactions on Neural Networks, vol. 17, no. 6, pp. 1646-1651, 2006.

[38] Y. Liu, Z. Wang, J. Liang, and X. Liu, "Stability and synchronization of discrete-time Markovian jumping neural networks with mixed mode-dependent time delays," IEEE Transactions on Neural Networks, vol. 20, no. 7, pp. 1102-1116, 2009. 
[39] P. Balasubramaniam and G. Nagamani, "Global robust passivity analysis for stochastic interval neural networks with interval time-varying delays and Markovian jumping parameters," Journal of Optimization Theory and Applications, vol. 149, no. 1, pp. 197-215, 2011.

[40] Z. Wang, H. Gao, J. Cao, and X. Liu, "On delayed genetic regulatory networks with polytopic uncertainties: robust stability analysis," IEEE Transactions on Nanobioscience, vol. 7, no. 2, pp. 154-163, 2008. 


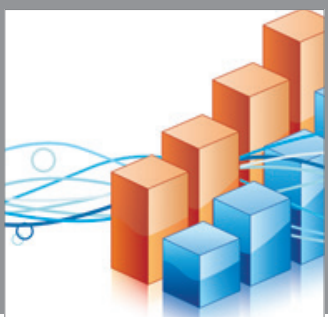

Advances in

Operations Research

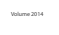

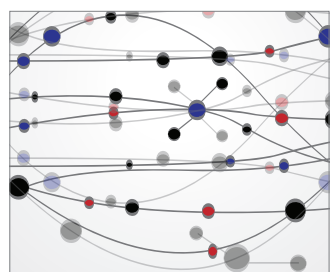

\section{The Scientific} World Journal
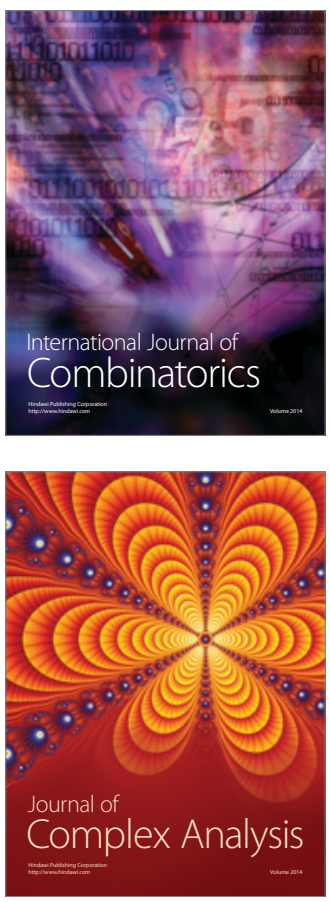

International Journal of

Mathematics and

Mathematical

Sciences
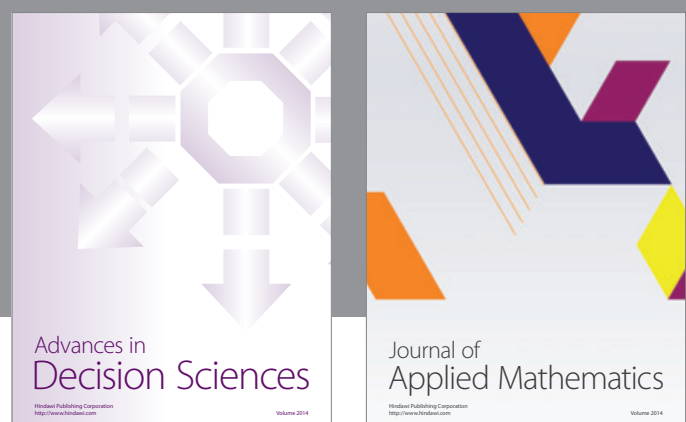

Journal of

Applied Mathematics
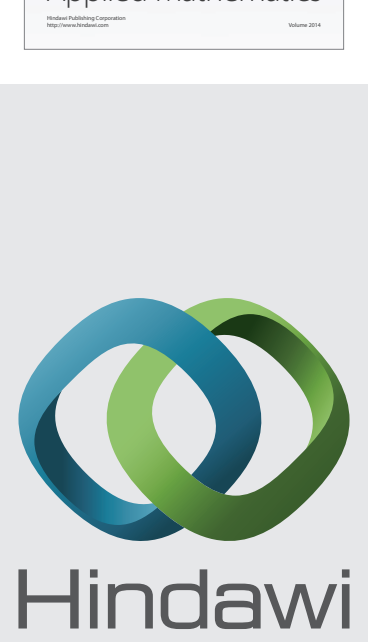

Submit your manuscripts at http://www.hindawi.com
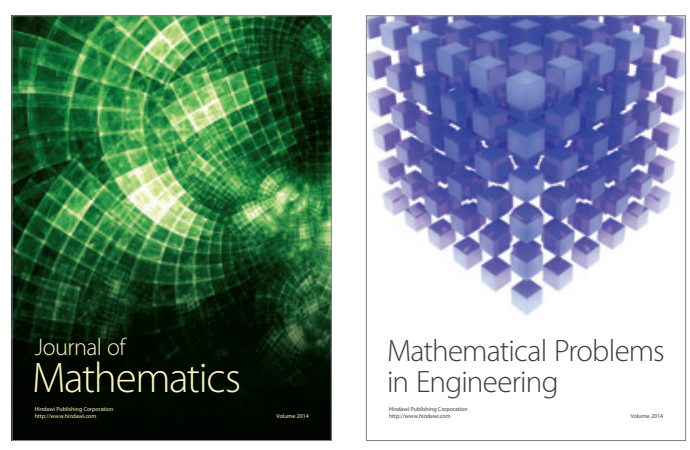

Mathematical Problems in Engineering
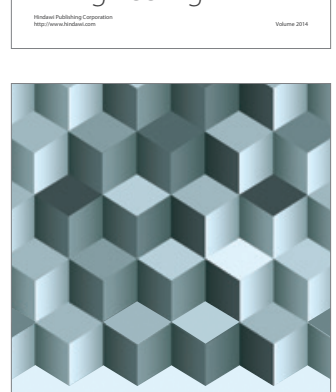

Journal of

Function Spaces
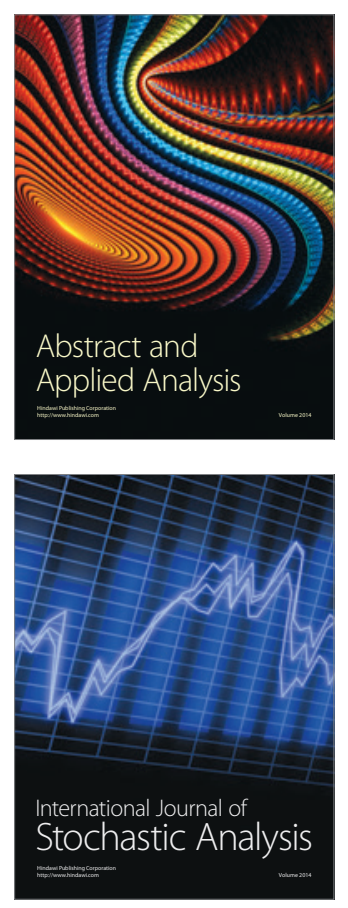

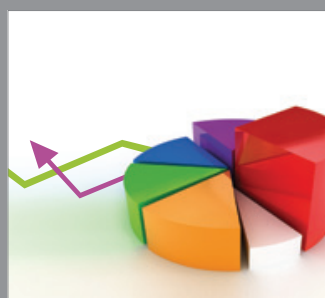

ournal of

Probability and Statistics

Promensencen
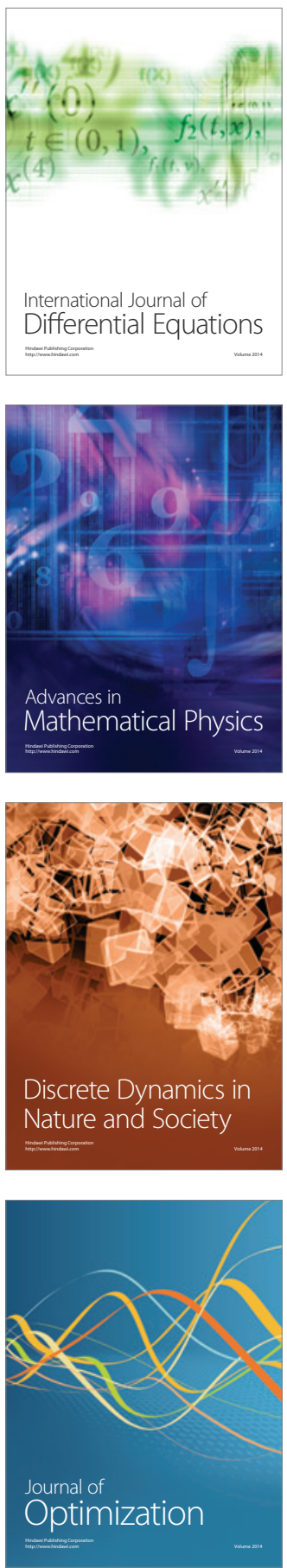